interactions involve three individuals: a donor, a recipient and a bystander. The donor uses a strategy to decide whether or not to cooperate and pay a cost that produces a benefit for the recipient. The bystander witnesses this and, using a rule termed a norm, assigns a reputation to the donor that is communicated to others in the population. In future social interactions, this reputation affects whether the donor receives the benefits of cooperation when taking on the role of a recipient.

One version of this interaction is known as a first-order system. In this scenario, two strategies exist. The donor can cooperate or not cooperate (defect). The bystander considers the donor's cooperation or defection when using a norm to assign a good or bad reputation.

Yet even in this simple system, four possible norms exist for the bystander: always assign a good reputation; always assign a bad reputation; assign a good reputation if the donor cooperates and a bad reputation if the donor defects; or assign a bad reputation if the donor cooperates and a good reputation if the donor defects. These norms vary in complexity. The first two are independent of the donor's action and the complexity is low. The latter two norms are dependent on the donor's action and the complexity is relatively high.

This reflects a general pattern. Give a bystander some information, and the level of complexity can vary between the possible norms. Moreover, the complexity of the mostcomplex norms increases with the information available, and the scope for increasing complexity is striking. In a second-order system, another component is added to the interaction. For example, both the donor and the bystander consider the reputation of the recipient. This allows 4 possible strategies and 16 possible norms. A third-order system could also include the donor's reputation, yielding 16 possible strategies and 256 possible norms ${ }^{5}$.

Santos and colleagues' fourth-order system additionally allows individuals to consider information about the past reputation of either the recipient or the donor. By incorporating the past, a donor's reputation is not dependent on a single point in time. In this scenario, 256 strategies and a staggering 65,536 norms are possible.

With ample scope for complexity in place, Santos and colleagues then examined each norm separately, and allowed the strategies used to evolve (the frequency of use of each strategy could change over time). The strategies that prevail, given a particular norm, affect the amount of cooperation that occurs. One norm, termed stern judging, stands out from the glut of conceivable norms as a relatively low-complexity norm that is highly likely to promote the evolution of cooperation.

The essence of stern judging is to assign a good reputation to a donor who cooperates with a good recipient or who defects with a bad recipient, and assign a bad reputation to a donor who defects with a good recipient or who cooperates with a bad recipient (Fig. 1). This is a simple second-order norm that supports the evolution of simple and highly cooperative strategies, and it does so even when tested in higher-order systems. From the profusion of feasible norms, more-complex norms do not improve the evolution of cooperation, at least up to the fourthorder system studied by the authors. This suggests that a relatively simple norm, with its correspondingly simple requirements in terms of processing and disseminating information, can suffice to drive indirect reciprocity.

This finding also raises a question for the future. Given so many conceivable norms, why use stern judging? In Santos and colleagues' system, strategies evolve, but norms do not. In reality, strategies and norms evolve together ${ }^{6}$. Both the way people behave (strategies) and the way they evaluate behaviour (norms) change over time, and this process almost certainly involves both genetic and cultural components ${ }^{7}$. Examining the co-evolution of strategies and norms with culture in the mix would be challenging in a fourth-order system, but it would increase our understanding of whether and when we might expect to observe people using reciprocity norms effectively to support cooperation.

In addition, in Santos and colleagues' work, every bystander in a given simulated population uses the same norm. However, in many social settings, there can be variation in the level of subtlety with which different people evaluate social situations. This kind of variation, which could result in bystanders using norms of different levels of complexity, may or may not ${ }^{8}$ result in disagreements between individuals about how to assign reputations. If disagreements occur, how much disagreement can indirect reciprocity tolerate before cooperation breaks down?

Finally, large-scale cooperation occurs in human societies ${ }^{9}$, and efforts to explain how this evolved have generated controversy, possibly because mutually compatible mechanisms are sometimes treated as strict alternatives. Perhaps the next step needed to address this will be to systematically combine multiple mechanisms ${ }^{4}$, including indirect reciprocity, and to test whether specific combinations of mechanisms are especially potent at promoting the evolution of cooperation.

Charles Efferson is in the Department of Psychology, Royal Holloway, University of London, Egham TW20 OEX, UK.

Ernst Fehr is in the Department of Economics, University of Zurich, Zurich 8006, Switzerland. e-mails: charles.efferson@rhul.ac.uk; ernst.fehr@econ.uzh.ch

1. Henrich, J. J. Econ. Behav. Org. 53, 3-35 (2004).

2. Santos, F. P., Santos, F. C. \& Pacheco, J. M. Nature 555, 242-245 (2018)

3. Alexander, R. D. The Biology of Moral Systems (Routledge, 1987).

4. van Veelen, M., García, J., Rand, D. G. \& Nowak, M. A. Proc. Natl Acad. Sci. USA 109, 9929-9934 (2012).

5. Ohtsuki, H. \& Iwasa, Y. J. Theor. Biol. 239, 435-444 (2006).

6. Pacheco, J. M., Santos, F. C. \& Chalub, F. A. C. C. PLoS Comput. Biol. 2, e178 (2006).

7. Richerson, P. J. \& Boyd, R. Not by Genes Alone: How Culture Transformed Human Evolution (Univ. Chicago Press, 2005).

8. Uchida, S. \& Sigmund, K. J. Theor. Biol. 263, 13-19 (2010).

9. Richerson, P. et al. Behav. Brain Sci. 39, e30 (2016).

\title{
A new era of rationally designed antipsychotics
}

The ideal drugs for treating schizophrenia are postulated to selectively block the D2 dopamine receptor with optimum binding kinetics. The structure of D2 bound to an antipsychotic sheds light on how to design such drugs. SEE LETTER P.269

\section{DAVID R. SIBLEY \& LEI SHI}

S chizophrenia is a disorder that involves hallucinations, delusions and cognitive impairment, and that affects nearly $1 \%$ of the global population ${ }^{1}$. The mainstays of therapy have been drugs that block the activity of the D2 dopamine receptor (D2R), a member of the large G-protein-coupled receptor (GPCR) superfamily of membrane proteins. Unfortunately, most of these antipsychotic drugs come with a plethora of debilitating side effects, many of which are due to off-target interactions with other GPCRs. On page 269, Wang et al. ${ }^{2}$ now report the crystal structure of D2R in complex with the antipsychotic drug risperidone. The structure reveals features that might be useful for the design or discovery of drugs that have greater selectivity for D2R than existing therapeutics, and consequently have fewer side effects.

The naturally occurring ligand for D2R is 

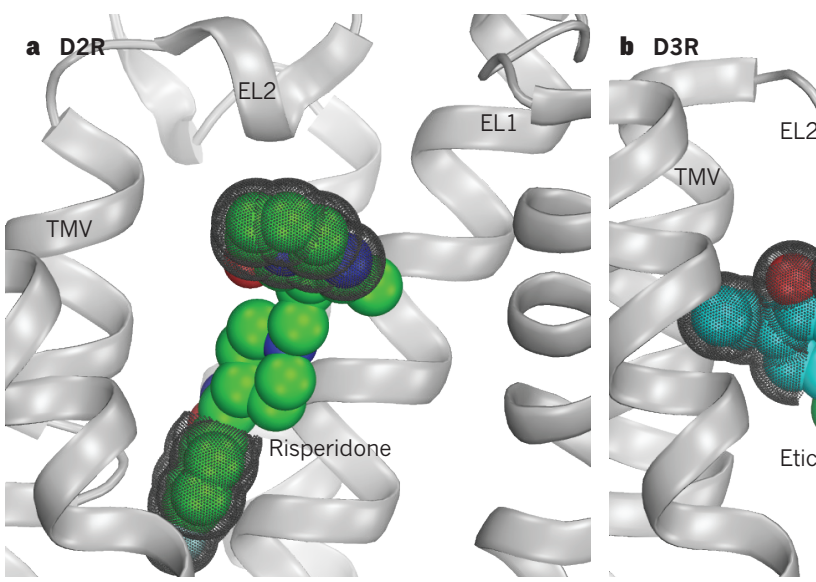

Figure 1 | Binding sites within crystal structures of D2-like receptors in complex with drug molecules. Drugs that block the activity of the D2 dopamine receptor (D2R) are used to treat schizophrenia, but also block the closely related D3 and D4 receptors (D3R and D4R), and exhibit debilitating side effects due, in part, to their interactions with other receptors. a, Wang et al. ${ }^{2}$ report the crystal structure of D2R in complex with the antipsychotic drug risperidone. They observe structural features and drug-receptor binding interactions not observed in the previously reported structure of D3R

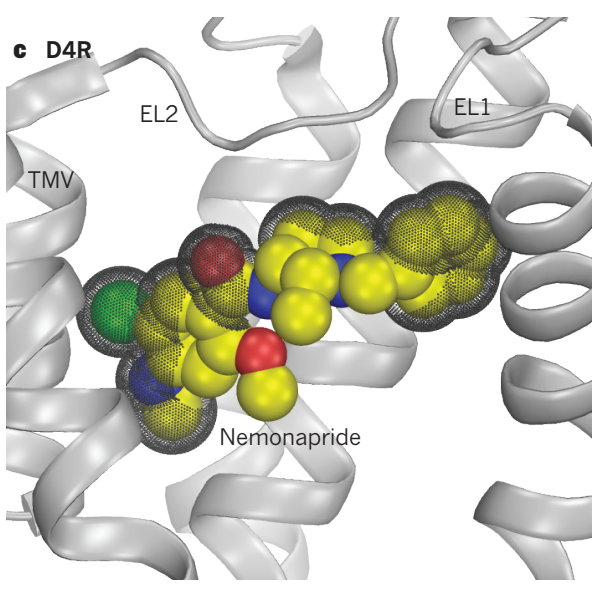

with the drug eticlopride ${ }^{11}$ (b), or of D4R with nemonapride ${ }^{9}$ (c). The drug molecules are shown as coloured space-filling structures, and the regions enclosed by dots make receptor contacts that are unique to each receptor. The identification of these contacts might help receptor-specific binding pockets to be delineated, which would aid the rational design of receptorselective drugs. Receptors are shown in grey; thick ribbons are $\alpha$-helices; thin regions are unstructured. EL1 and EL2 are extracellular loops. TMV is a transmembrane-spanning segment. a neurotransmitter called dopamine, which mediates various physiological functions, including the control of coordinated movement, cognition and the reinforcing properties of drugs of abuse. There are five receptors for dopamine, which fall into two subgroups on the basis of their associated intracellular signalling pathways and their affinities for various drugs ${ }^{3}$ : D1-like receptors (D1R and D5R) and D2-like receptors (D2R, D3R and D4R). As early as the 1970s, it was hypothesized that the therapeutic effects of antipsychotic drugs were due to them blocking D2-like, rather than D1-like, receptors ${ }^{4,5}$, but the existence of multiple D2-like receptors was not discovered until they were cloned some 15 years later ${ }^{6}$.

Although it has been proposed that antipsychotic-drug action might involve the blocking of D3R or D4R, it is now generally agreed that $\mathrm{D} 2 \mathrm{R}$ blockade is necessary, and probably sufficient, for the amelioration of the 'positive' symptoms of schizophrenia, such as delusions, hallucinations and disordered thinking $^{7}$. (Antipsychotics currently in use are less effective at treating the 'negative' symptoms of this disorder, which include social withdrawal and cognitive impairment.) Progress has been made in the development of D3R-selective ${ }^{8}$ and D4R-selective ${ }^{9}$ compounds, but there remains a paucity of drugs with high selectivity for the closely related D2R (ref. 10), despite its clear therapeutic importance.

Crystal structures of D3R bound to the drug eticlopride ${ }^{11}$ and of D4R bound to the antipsychotic nemonapride ${ }^{9}$ have previously been reported. Wang and colleagues' structure now reveals that risperidone interacts with D2R in a different way from how eticlopride and nemonapride interact with D3R and D4R (Fig. 1). One part of risperidone (known as a benzisoxazole group) extends below the orthosteric site (the site at which dopamine binds) in D2R, and penetrates deep into a hydrophobic pocket that is not formed in the D3R and D4R structures. A second, extended binding pocket above the orthosteric site in D2R encloses another part of risperidone (a tetrahydropyridopyrimidinone group). This pocket consists of amino-acid residues from extracellular loop 1 (EL1) and three transmembrane helices (TMIII, TMVI and TMVII).

Strikingly, in D2R, a residue within another extracellular loop (EL2), and which is immediately adjacent to an evolutionarily conserved cysteine residue, is buried within the protein and faces the fourth transmembrane helix (TMIV). By contrast, the equivalent residues in D3R and D4R are oriented towards water in the extracellular milieu. EL2 therefore forms a short helical segment in D2R, but is largely extended and unstructured in D3R and D4R (Fig. 1). Consequently, the structural configurations near the EL1 and EL2 interface in D3R and D4R are different from those in D2R.

Wang et al. propose that such divergence contributes to the formation of distinct, extended binding pockets in these three receptors, as has been previously suggested ${ }^{9,11,12}$. Drugs designed to selectively engage the distinctive pockets in the D2R structure might display enhanced D2R selectivity. Analogous structure-based drug-discovery efforts have already proved useful in identifying highaffinity compounds ${ }^{13}$ that block D3R (ref. 14) or that activate D4R (ref. 9).

Notably, the receptor segments directly above the risperidone-binding site in D2R form a hydrophobic 'patch' composed of the side chains of three amino-acid residues, designated Leu $94^{2.64}, \operatorname{Trp} 100^{\mathrm{EL} 1}$ and Ile $184^{\mathrm{EL} 2}$. This patch potentially restricts the access of molecules to the D2R binding pocket. Wang and co-workers hypothesized that this feature might regulate the dissociation of risperidone from the D2R binding site, and thus affect its residence time at the receptor.

The authors tested this hypothesis by mutating single residues in the patch and by making a mutant $\mathrm{D} 2 \mathrm{R}$ in which both Ile $184^{\mathrm{EL} 2}$ and Leu $94^{2.64}$ were replaced. These mutations dramatically reduced risperidone's residence time from 233 minutes in the wildtype receptor to as little as 6 minutes in the double mutant. This effect is notable because the kinetics of antipsychotic-drug binding to D2R might correlate with a tendency to produce debilitating extrapyramidal side effects (EPS), which include rigidity, tremors and involuntary movements. Antipsychotic drugs that cause fewer EPS, such as risperidone, are said to be atypical, and it has been suggested that antipsychotics with shorter D2R residence times exhibit greater 'atypicality ${ }^{15,16}$. Shorter residence times at D2R might enable a minimum level of dopaminergic stimulation, which lessens EPS. The current findings illustrate how elements of the D2R structure can regulate the kinetics of drug binding, which in turn might be associated with desirable therapeutic outcomes.

The hydrophobic patch in D2R is absent in the D3R and D4R structures, presumably because of the separation between the analogous EL1 and EL2 residues in the latter two receptors. Thus, an intriguing question is whether the kinetics of drug binding to D2R are fundamentally different from those to D3R and D4R, particularly for molecules that have similar affinities for the three receptors. In other words, are the kinetics of drug binding to these receptors patch-dependent?

Of further interest is the observation ${ }^{17}$ that risperidone is not selective between D2R, D3R and $\mathrm{D} 4 \mathrm{R}$, thus raising the question of how this 
drug can bind differently to these receptors and still have identical affinities for them. Additional structures (such as D3R or D4R in complex with risperidone) will probably be needed to answer this. Nonetheless, we expect that Wang and colleagues' D2R-risperidone structure, along with the previous D3R and D4R structures, will accelerate the design and discovery of D2R ligands that have higher selectivity than current antipsychotics, and potentially greater therapeutic impact.

David R. Sibley is in the Molecular Neuropharmacology Section, National Institute of Neurological Disorders \& Stroke,
National Institutes of Health, Bethesda, Maryland 20892-3723, USA. Lei Shi is in the Computational Chemistry and Molecular Biophysics Unit, National Institute on Drug Abuse, National Institutes of Health, Baltimore, Maryland 21224, USA. e-mails: sibleyd@ninds.nih.gov; lei.shi2@nih.gov

1. Perala, J. et al. Arch. Gen. Psychiatry 64, 19-28 (2007).

2. Wang, S. et al. Nature 555, 269-273 (2018).

3. Kebabian, J. W. \& Calne, D. B. Nature 277, 93-96 (1979).

4. Creese, I., Burt, D. R. \& Snyder, S. H. Science 192 , 481-483 (1976)

5. Seeman, P., Lee, T., Chau-Wong, M. \& Wong, K. Nature 262, 717-719 (1976)

6. Sibley, D. R. \& Monsma, F. J. Jr Trends Pharmacol. Sci. 13, 61-69 (1992)
7. Kapur, S. \& Remington, G. Biol. Psychiatry 50, 873-883 (2001).

8. Keck, T. M., Burzynski, C., Shi, L. \& Newman, A. H. Adv. Pharmacol. 69, 267-300 (2014).

9. Wang, S. et al. Science 358, 381-386 (2017).

10.Moritz, A. E., Free, R. B. \& Sibley, D. R. Cell. Signal. 41, 75-81 (2018)

11.Chien, E. Y. et al. Science 330, 1091-1095 (2010).

12.Newman, A. H. et al. J. Med. Chem. 55, 6689-6699 (2012).

13.Löber, S., Hübner, H., Tschammer, N. \& Gmeiner, P. Trends Pharmacol. Sci. 32, 148-157 (2011)

14.Carlsson, J. et al. Nature Chem. Biol. 7, 769-778 (2011).

15.Kapur, S. \& Seeman, P. Am. J. Psychiatry 158, 360-369 (2001).

16.Sykes, D. A. et al. Nature Commun. 8, 763 (2017).

17.Seeman, P. Clin. Neurosci. Res. 1, 53-60 (2001).

This article was published online on 26 February 2018.

\section{Transistors driven by superconductors}

\section{A hybrid transistor device has been made in which a superconductor forms a seamless interface with a semiconductor. The study of such interfaces could open the way to innovative applications in electronics. SEE ARTICLE P.183}

\section{YOSHIHARU KROCKENBERGER \& YOSHITAKA TANIYASU}

I ntegrating superconductors with semiconductors has long been thought to be essential to overcome the current limitations of electronic devices, but has been challenging to achieve. On page 183, Yan et al. ${ }^{1}$ report their use of a technique known as molecular beam epitaxy to grow layers of semiconductors on top of a superconductor. The resulting device has potentially useful electronic properties that hint at future applications for semiconductor-superconductor interfaces.

The development of increasingly sophisticated electronic devices is aided by efforts to make new combinations of materials - or, more specifically, new interfaces between materials, at which potentially useful electronic effects can occur. The credo underlying this concept is that "the interface is the

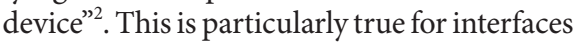
involving superconductors.

For example, Josephson junctions consist of two superconductors separated by a thin barrier, such as an insulator or a nonsuperconducting metal. Cooper pairs of electrons - the bound electron pairs that are responsible for superconductivity - can tunnel across the barrier in a fascinating physical process that has led to the development of devices such as those that mix or emit light at terahertz frequencies ${ }^{3}$. Interfacing superconductors with semiconductors ${ }^{4}$ such as indium arsenide (an arsenic-based material) can trigger Andreev reflection processes in which a normal electric current becomes a superconducting current. And if a ferromagnetic material (a material that exhibits the type of magnetism associated with iron) is used as the barrier in a Josephson junction, even more opportunities emerge for the manipulation of controllable electronic states $^{5}$.

Yan and colleagues now report the synthesis of interfaces formed between two a

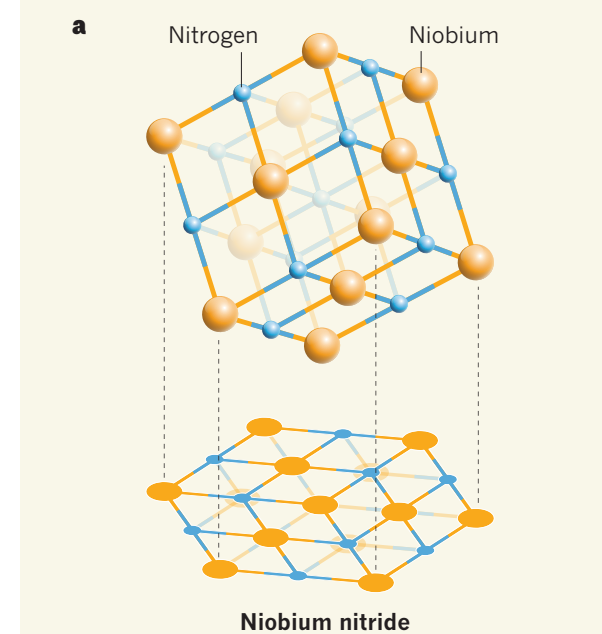

nitrides (nitrogen-containing materials), one a superconductor and the other a semiconductor. Nitride semiconductors ${ }^{6}$ are non-toxic, which makes them much more desirable for most applications than toxic arsenic-containing semiconductors. They can be synthesized in well-established procedures using molecular beam epitaxy - a technique in which atomized elements are deposited on a substrate in a vacuum to form thin films of single crystals. Nitride superconductors are also non-toxic, and, more importantly, are highly stable, particularly in ambient conditions (unlike many superconductors). The authors demonstrate that they can fabricate interfaces between a nitride superconductor and devices known as high-electron-mobility transistors ${ }^{7}$ (HEMTs) made from nitride semiconductors. HEMTs are widely used in communications infrastructures.

One problem that Yan and colleagues had to contend with is the fact that their nitride semiconductors have hexagonal crystal lattices, whereas the superconductor

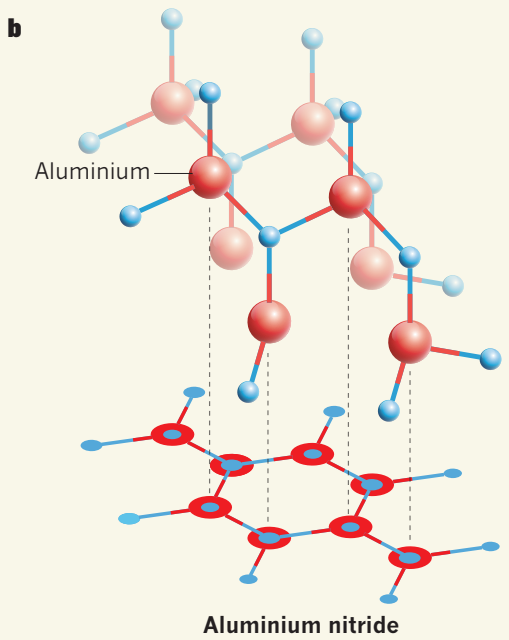

Figure 1 | Aligned views of materials that have different crystal lattices. a, The crystal lattice of the superconductor niobium nitride is cubic, but looks hexagonal when viewed from a particular orientation. b. The crystal lattice of the semiconductor aluminium nitride is hexagonal, and can therefore be aligned with the hexagonal arrangement shown in a. This allowed Yan et al. ${ }^{1}$ to prepare electronic devices in which a thin film of aluminium nitride is grown on top of niobium nitride, and the atoms of the two materials are aligned at the interface. 